\title{
Prediction of adverse effects of preeclampsia
}

\author{
Khushboo Tongaria ${ }^{1 *}$, Ashok Kumar², Simar Kaur ${ }^{3}$
}

\begin{abstract}
${ }^{1}$ Department of Obstetrics and Gynaecology, ESIC Medical college and Hospital, Faridabad, Haryana, India ${ }^{2}$ Department of Obstetrics and Gynaecology, Maulana Azad Medical College and Associated Hospital, New Delhi, India

${ }^{3}$ Department of Obstetrics and Gynaecology, Akash Hospital, New Delhi, India
\end{abstract}

Received: 08 May 2020

Accepted: 29 September 2020

\author{
*Correspondence: \\ Dr. Khushboo Tongaria, \\ E-mail drktong91@gmail.com
}

Copyright: () the author(s), publisher and licensee Medip Academy. This is an open-access article distributed under the terms of the Creative Commons Attribution Non-Commercial License, which permits unrestricted non-commercial use, distribution, and reproduction in any medium, provided the original work is properly cited.

\section{ABSTRACT}

Background: To predict the adverse maternal, perinatal and combined (both maternal and perinatal) outcome in preeclampsia by using various clinical and laboratory variables.

Methods: Five hundred fifty women diagnosed with preeclampsia were included and twenty-four women were excluded from the study due to exclusion criteria, six women decline to participate, twenty women were lost to follow up, three women withdrew consent, so a total of 497 women were followed up in the study.

Results: Mean age of study population was $26.82 \pm 4.48$ years. Majority of women with preeclampsia delivered vaginally. Forty-five $(9.05 \%)$ developed neurological complication. Mean gestational age at delivery (weeks) in patients who developed adverse outcome was $34.58 \pm 3.74$ weeks and in patients with normal outcome is $38.62 \pm 1.59$ weeks. Mean birth weight of newborns were $2.1 \pm 0.73 \mathrm{~kg}$ and $1.85 \pm 0.61 \mathrm{~kg}$ for newborns with adverse outcomes. Majority of perinatal complication was small for gestational age 267 (54.37\%) followed by prematurity $262(53.36 \%)$. Total number of adverse perinatal events was six hundred seventy-seven as multiple neonates had more than one perinatal outcome. In combined (both maternal and perinatal) adverse outcome-374 (75\%) developed adverse outcome, 123 (25\%) developed normal pregnancy outcome.

Conclusion: This study found out simple clinical, biochemical tools for monitoring pregnant women and accurately identifying who was at greatest risk of severe complications. By identifying those women at highest risk of adverse maternal outcomes well before that outcome occurs, transportation and treatment can be targeted to those women most in need. This clinical prediction tool found to be an important contributor as it offers the potential to improve health outcomes of women for a condition that is at the root of a large amount of morbidity and mortality in the developing world.

Keywords: Prediction, Preeclampsia, Adverse effects

\section{INTRODUCTION}

Preeclampsia remains a leading cause of maternal mortality in India and it complicates approximately 4 to $6 \%$ of all pregnancies and a major cause of maternal, fetal and neonatal morbidity and mortality, contributing a significant healthcare economic burden. ${ }^{1}$ The disease not only affects pregnancy outcome, but also predisposes mother and child to long-term health complications such as cardiovascular disease.
Preeclampsia is a placental specific disorder that becomes clinically apparent after 20 weeks of gestation or within the first 4-6 weeks postpartum. Preeclampsia can affect both the mother and the unborn baby and is estimated to affect between 5 and $8 \%$ of healthy pregnancies. ${ }^{2}$ It is responsible for about 76,000 maternal deaths and 5,00,000 infant deaths per year. In about $30 \%$ of patients with preeclampsia, placental insufficiency or reduced placental blood flow causes intrauterine growth restriction or fetal death. ${ }^{3}$ WHO 
estimates that in developing countries, preeclampsia and eclampsia account for $25 \%$ of stillbirths, $25 \%$ of neonatal deaths, and an unknown number of long-term neurological disabilities. ${ }^{4}$

There is a need to demystify disease at community and facility levels so women get proper care. Preeclampsia is caused by maternal endothelial dysfunction from aberrant placentation. With no effective or affordable large-scale screening tests to predict the adverse outcome of preeclampsia, the present study was conducted to study relation between various clinical and laboratory variables and occurrence of adverse maternal and neonatal outcomes in patients with preeclampsia.

\section{METHODS}

This prospective observational study was conducted in the department of obstetrics and gynaecology, Maulana Azad medical college and associated Lok Nayak GIPMER and GNEC hospital, New Delhi for 18 months (October 2016-April 2018). 550 women diagnosed with preeclampsia were included and 24 were excluded from study due to exclusion criteria (chronic hypertension, pre-existing preeclampsia, twin gestation). 6 women declined to participate, twenty women were lost to follow up, 3 women withdrew consent, so finally a total of 497 women were followed up.

Adverse outcome included viz. maternal outcome antepartum eclampsia, postpartum eclampsia, posterior reversible encephalopathy syndrome and Stroke. Similarly, cardiovascular adverse outcome included DCMP (Dilated cardiomyopathy), myocardial infarction and Pulmonary oedema. With regard to haematological adverse outcome-transfusion of any blood product and platelet count $<50 \times 10^{3}$ per/l. Various other outcome such as HELLP, acute renal insufficiency, abruption, fundal changes and maternal mortality were also included.

Perinatal outcome included still birth, neonatal death, low birth weight, small for gestational age, appropriate for gestational age, respiratory distress syndrome and Prematurity.

All women presented with preeclampsia were included in study. Ethical clearance was obtained from institutional ethics committee. Written informed consent was taken prior from pregnant women willing for enrolment in study. All pregnant women who fulfil the criteria of study were enrolled and evaluated on basis of predesigned and pretested standard proforma which included patient information.

\section{History and examination}

A detailed history which included presenting symptoms such as headache, nausea, vomiting, blurring of vision, epigastric pain, chest pain and dyspnoea. Blood pressure readings were taken along with assessment of proteinuria to confirm eligibility of patient for study. $\mathrm{SpO}_{2}$ was checked for all patients. The eligible patients were subjected to various haematological and biochemical investigations.

\section{Clinical laboratory tests}

Haematology: Haemoglobin, haematocrit, platelet Count, International Normalised Ratio, activated partial thromboplastin time (APTT).

Hepatic and renal: Liver and kidney function testSGOT-Serum glutamate oxaloacetate transaminase, SGPT-Serum glutamate pyruvate transaminase, Alkaline Phosphatase (ALP), total bilirubin, total protein, serum albumin, blood urea and serum creatinine, serum uric acid, serum lactate dehydrogenase, urine for albumin by dipstick method, 24-hour urine for protein.

Fetal surveillance: Cardiotocography, ultrasound for assessment of fetal weight, amniotic fluid volume and umbilical artery doppler. Worst value (e.g. highest systolic blood pressure or lowest platelet count or highest creatinine) measured after admission and before outcome occurrence were used. Any interventions done after admission such as administration of antihypertensives, magnesium sulphate or steroids as per hospital protocol after admission were noted. All subjects were followed up for occurrence of adverse maternal and perinatal outcome.

\section{Statistical analysis}

SPSS version 16 was used for statistical analysis. The quantitative variables were expressed in terms of mean \pm SD and compared by using student t-test (unpaired). Qualitative variables were expressed as frequency (\%) and analysed by using chi square test. ROC (receiver operating curve) was made to identify the threshold value for various parameters to detect adverse maternal and perinatal and both outcomes. Variables with a statistically significant association $(p<0.05)$ with the combined adverse maternal outcomes were analysed by using multivariable regression model for prediction.

\section{RESULTS}

The mean age of patients in study group was $26.8 \pm 4.48$ years and mean period of gestation at time of delivery was 35.6 \pm 3.76 weeks. More than half of study population was multigravida $(61.97 \%)$. Among 308 multigravida women with preeclampsia, only $12(3.8 \%)$ had hypertensive disorder in previous pregnancy. Among 497 women with preeclampsia, 132 (26.55\%) had gestational hypertension in present pregnancy, 28 $(5.6 \%)$ had gestational diabetes in present pregnancy, 3 $(0.6 \%)$ had type 2 diabetes. Maximum number of study population were Muslim (58.14\%) and other half were 
Hindu religion (41.86\%). Most of the women with preeclampsia belong to lower upper class (40.64\%) according to modified Kuppuswamy scale followed by lower class. Under interventions as per standard protocol, $492(98.99 \%)$ study population required antihypertensives for blood pressure control, 304 $(61.17 \%)$ required magnesium sulphate, 161 (32.39\%) study population required steroid for fetal lung maturity. Out of 497 study population-majority of women with preeclampsia delivered vaginally. Normal vaginal delivery 298 (60.69\%), Instrumental delivery (forceps and vacuum) $9(1.83 \%)$ and one third of women required caesarean section for fetal or maternal indication $184(37.47 \%)$.

Out of 497 study population, $45(9.05 \%)$ developed neurological complication under which 36 (7.24\%) developed antepartum eclampsia, $5(1.00 \%)$ developed postpartum eclampsia, $3(0.64 \%)$ developed posterior reversible encephalopathy syndrome and 1 (0.22\%) developed stroke. Majority of complication included alteration in haematological $75(15.09 \%)$ system in which $74(14.88 \%)$ patients required transfusion of any blood product and $1(0.22 \%)$ had platelet count less than 50,000/L managed without any transfusion. 20 $(0.425 \%)$ patients with preeclampsia complained of bleeding per vaginum were diagnosed with abruption. 9 $(1.81 \%)$ women with preeclampsia had serious complications like pulmonary oedema and $3(0.60 \%)$ had DCMP. In the present study mean gestational age at delivery in patients who developed adverse outcome was $34.58 \pm 3.74$ weeks and in patients with normal outcome was $38.62 \pm 1.59$ weeks. Out of 497 study population, 6 patients were undelivered due to maternal mortality in antenatal period, hence total 491 babies were evaluated for perinatal outcomes. Mean birth weight of newborns was $2.1 \pm 0.73 \mathrm{~kg}$ and $1.85 \pm 0.61 \mathrm{~kg}$ for newborns with adverse outcomes. Out of 491, 169 (34\%) newborns birth weight was between 2.5 to $4 \mathrm{~kg}$ and $39(10.89 \%)$ developed adverse outcome. 138 (27.7\%) newborns birth weight was between 2 to $2.5 \mathrm{~kg}$ and $136(37.98 \%)$ developed adverse outcome.

\section{Maternal outcomes}

Out of 497 patients followed up till delivery, 147 (29.58\%) developed various adverse maternal outcomes like pulmonary oedema, eclampsia, placental abruption. Most common complication among patients with preeclampsia had alteration in haematological system and required transfusion. Out of 147 women, 75 (51.02\%) women needed transfusion of blood or blood products due to fall in haematocrit or platelet count. 41 (27.89\%) women developed eclampsia among which 36 $(87.80 \%)$ were antepartum and $5(12.19 \%)$ were postpartum. $9(6.12 \%)$ patients admitted with severe preeclampsia developed pulmonary oedema. Out of which 3 required ionotropic and ventilator support. Twenty (13.60\%) women developed abruption and 9 (6.12\%) developed HELLP syndrome and required urgent intervention, transfusion of blood and blood products and termination of pregnancy. A total of 19 $(12.92 \%)$ patients in the study population had mortality due to congestive cardiac failure $(n=7)$, sepsis with multiorgan dysfunction $(\mathrm{n}=5)$, acute kidney injury $(n=3)$, disseminated intravascular coagulation and septic shock $(n=4)$. All patients admitted with preeclampsia were subjected to various investigations at admission. Headache was the most common symptom among patients who presented with preeclampsia, seen in 171 (34.4\%) patients. Among women who developed adverse maternal outcome, $46.94 \%$ had complains of headache compared to $29.14 \%$ patients who had a normal maternal outcome. This observation was statistically significant $(\mathrm{p}<0.05)$. Other statistically significant presenting symptom was chest pain/dyspnoea. Symptoms of nausea, vomiting, blurring of vision, epigastric pain were not found to be significant statistically (Table 1-3).

\section{Perinatal outcomes}

Adverse perinatal outcomes were seen in $73.3 \%$ of babies. This included prematurity, small for gestational age foetuses, stillbirths etc. 6 babies were excluded from further analysis of perinatal outcomes because their mothers died during the antepartum period due to various complications of preeclampsia. 6 babies out of 497 were undelivered as their mothers died during antepartum period. Hence, statistical analysis for perinatal outcomes was done for 491 babies. Most common perinatal complications were small for gestational age in $267(54.37 \%)$ followed by prematurity in $262(53.36 \%)$, still birth in $68(13.84 \%)$ and respiratory distress syndrome in 59 (12.01\%) of study population. Mean birth weight of babies with adverse perinatal outcomes was $1.85 \pm 0.61 \mathrm{~kg}$, which was $1.1 \mathrm{~kg}$ less than mean birth weight of babies with normal perinatal outcomes. 52\% babies in study population were born preterm due to pregnancy complicated with preeclampsia. Among 147 (29.95\%) babies born at or beyond 37 weeks of gestation 81 (22.62\%) had an adverse outcome such as stillbirths, neonatal deaths, small for gestational age. 267 (54.37\%) babies were small for gestational age, in which 56 $(20.97 \%)$ were between 39-41 weeks and 54 (20.97\%) were between 28-34 weeks. Headache was the most common symptom among patients who presented with preeclampsia, seen in $171(34.4 \%)$ patients. Among women who developed adverse maternal outcome, $39.56 \%$ had complains of headache compared to $20.30 \%$ patients who had a normal maternal outcome. This observation was statistically significant $(p<0.05)$. Other statistically significant presenting symptom was nausea and vomiting. 
Table 1: Comparative analysis of various parameters among study population with or without adverse outcome.

\begin{tabular}{|c|c|c|c|}
\hline Characteristics & $\begin{array}{l}\text { Study population } \\
\text { with adverse } \\
\text { outcome }(n=147)\end{array}$ & $\begin{array}{l}\text { Study population } \\
\text { with normal } \\
\text { outcome }(n=350)\end{array}$ & P value \\
\hline Mean age $($ years $) \pm S D(n=497)$ & $27.11 \pm 4.78$ & $26.70 \pm 4.35$ & 0.179 \\
\hline $\begin{array}{l}\text { Mean gestational age at delivery }(\text { weeks }) \pm \text { SD } \\
(n=491)\end{array}$ & $34.58 \pm 3.74$ & $38.62 \pm 1.59$ & $<0.001 *$ \\
\hline Mean birth weight $(\mathrm{kg}) \pm \mathrm{SD}(\mathrm{n}=491)$ & $1.92 \pm 0.64$ & $2.24 \pm 0.75$ & $<0.001 *$ \\
\hline Primigravida $(n=189)$ & $59(31.2 \%)$ & $130(68.8 \%)$ & 0.394 \\
\hline Multigravida $(\mathrm{n}=308)$ & $88(28.57 \%)$ & $220(71.42 \%)$ & 0.394 \\
\hline Mean SBP (mmHg) & $159.92 \pm 18.09$ & $153.59 \pm 15.18$ & $<0.001^{*}$ \\
\hline Mean DBP (mmHg) & $103.41 \pm 8.95$ & $101.34 \pm 9.64$ & $0.013 *$ \\
\hline Mean MAP (mmHg) & $122.24 \pm 10.91$ & $118.76 \pm 10.75$ & $<0.001 *$ \\
\hline Mean $\mathrm{SpO}_{2}(\%)$ & $96.90 \pm 2.60$ & $97.80 \pm 1.18$ & $<0.001 *$ \\
\hline Hb $(g m / d l)(n=497)$ & $9.11 \pm 2.53$ & $10.85 \pm 1.71$ & $<0.001 *$ \\
\hline Het $(\%)(n=497)$ & $28.13 \pm 7.03$ & $32.54 \pm 5.04$ & $<0.001 *$ \\
\hline PIt $\left(\mathrm{lac} / \mathrm{mm}^{3}\right)(\mathrm{n}=497)$ & $1.51 \pm 0.96$ & $1.74 \pm 0.78$ & $0.002 *$ \\
\hline INR $(n=497)$ & $1.16 \pm 0.44$ & $1.00 \pm 0.21$ & $<0.001 *$ \\
\hline Urine albumin $(n=497)$ & $1.85 \pm 0.78$ & $1.56 \pm 0.73$ & $<0.001 *$ \\
\hline Urea $(\mathrm{mg} / \mathrm{dl})(\mathrm{n}=497)$ & $38.92 \pm 38$ & $28.96 \pm 16.49$ & $<0.001 *$ \\
\hline S. Cr (mg/dl) $(n=497)$ & $1.06 \pm 0.9$ & $0.83 \pm 0.7$ & $0.001 *$ \\
\hline SGOT $($ U/l) $(n=497)$ & $149.52 \pm 393.06$ & $53.82 \pm 85.71$ & $<0.001 *$ \\
\hline SGPT (U/I) (n=497) & $120.12 \pm 230.21$ & $55.84 \pm 85.66$ & $<0.001 *$ \\
\hline T. Bil (mg/dl) $(\mathrm{n}=497)$ & $1.10 \pm 1.62$ & $0.70 \pm 0.84$ & $<0.001 *$ \\
\hline Uric acid (mg/dl) $(n=164)$ & $6.99 \pm 2.19$ & $5.80 \pm 1.52$ & $<0.001 *$ \\
\hline LDH (U/I) $(n=207)$ & $662.38 \pm 415.88$ & $427.29 \pm 256.85$ & $<0.001 *$ \\
\hline T. Prt (mg/dl) $(n=177)$ & $5.95 \pm 1$ & $5.99 \pm 1.28$ & 0.429 \\
\hline
\end{tabular}

Table 2: Comparative analysis of various parameters of women with preeclampsia with adverse and normal perinatal outcome.

\begin{tabular}{|c|c|c|c|c|}
\hline \multirow{2}{*}{ Investigations } & \multirow{2}{*}{ No. of cases } & Adverse $(n=364)$ & Normal $(n=133)$ & \multirow{2}{*}{ P value } \\
\hline & & Mean士SD & Mean \pm SD & \\
\hline SBP (mmHg) & 497 & $157.46 \pm 17.33$ & $150.01 \pm 11.64$ & $<0.001 *$ \\
\hline DBP (mmHg) & 497 & $102.99 \pm 9.79$ & $99.11 \pm 7.94$ & $<0.001 *$ \\
\hline MAP (mmHg) & 497 & $121.14 \pm 11.41$ & $116.08 \pm 8.37$ & $<0.001^{*}$ \\
\hline $\mathrm{SpO}_{2}(\%)$ & 497 & $97.42 \pm 1.94$ & $97.84 \pm 1.14$ & $0.009 *$ \\
\hline Hb (gm/dl) & 497 & $10.22 \pm 2.22$ & $10.66 \pm 1.86$ & $0.021 *$ \\
\hline Het $(\%)$ & 497 & $31.02 \pm 6.16$ & $31.81 \pm 5.69$ & 0.098 \\
\hline PIt $\left(\mathrm{lac} / \mathrm{mm}^{3}\right)$ & 497 & $1.66 \pm 0.88$ & $1.72 \pm 0.74$ & 0.225 \\
\hline INR & 497 & $1.07 \pm 0.34$ & $1.01 \pm 0.18$ & $0.031 *$ \\
\hline Urine albumin & 497 & $1.75 \pm 0.78$ & $1.38 \pm 0.61$ & $<0.001 *$ \\
\hline Urea (mg/dl) & 497 & $33.69 \pm 28.72$ & $27.01 \pm 9.63$ & $0.004 *$ \\
\hline S. Cr (mg/dl) & 497 & $0.95 \pm 0.89$ & $0.77 \pm 0.19$ & $0.011 *$ \\
\hline SGOT (U/I) & 497 & $94.84 \pm 264.1$ & $47.34 \pm 63.32$ & $0.020 *$ \\
\hline SGPT (U/I) & 497 & $82.67 \pm 166.88$ & $53.44 \pm 63.62$ & $0.025^{*}$ \\
\hline T. Bil (mg/dl) & 497 & $0.87 \pm 1.31$ & $0.68 \pm 0.3$ & $0.047^{*}$ \\
\hline Uric acid (mg/dl) & 164 & $6.36 \pm 2$ & $5.72 \pm 1.22$ & $0.030 *$ \\
\hline LDH (U/I) & 207 & $543.96 \pm 360.47$ & $388.64 \pm 216.87$ & $0.003^{*}$ \\
\hline T. Prt (mg/dl) & 177 & $5.96 \pm 1.19$ & $6.01 \pm 1.27$ & 0.408 \\
\hline
\end{tabular}

SBP-systolic blood pressure, DBP-diastolic blood pressure, MAP-mean arterial pressure, mmHg-millimeter of mercury, SpO2saturation of peripheral oxygen, Hb-Haemoglobin, Hct-Haematocrit, Plt-platelet, INR-Internationalised normal ratio, SCr- Serum creatinine, SGOT-Serum glutamate oxaloacetate transaminase, SGPT-Serum glutamate pyruvate transaminase, T. Bil-total bilirubin, LDH-Lactate dehydrogenase, T Prt-Total protein, mg/dl-milligram per decilitre, U/l- unit per litre. 
Table 3: Comparative analysis of various findings at admission among women with preeclampsia who developed adverse outcomes (both maternal and paternal) and those with normal outcomes.

\begin{tabular}{|c|c|c|c|}
\hline \multirow{2}{*}{ Examination findings } & Adverse $(n=374)$ & Normal $(n=123)$ & \multirow{2}{*}{ P value } \\
\hline & Mean+SD & Mean+SD & \\
\hline SBP (mmHg) & $157.24 \pm 17.16$ & $150.07 \pm 12.06$ & $<0.001 *$ \\
\hline DBP (mmHg) & $102.85 \pm 9.71$ & $99.20 \pm 8.17$ & $<0.001 *$ \\
\hline MAP (mmHg) & $120.98 \pm 11.31$ & $116.16 \pm 8.67$ & $<0.001 *$ \\
\hline $\mathrm{SpO}_{2}(\mathrm{mmHg})$ & $97.42 \pm 1.94$ & $97.88 \pm 1.06$ & 0.082 \\
\hline Hb $(\mathrm{gm} / \mathrm{dl})$ & $10.13 \pm 2.27$ & $10.97 \pm 1.51$ & $<0.001 *$ \\
\hline Het $(\%)$ & $30.80 \pm 6.31$ & $32.57 \pm 4.92$ & $0.002 *$ \\
\hline Plt (lac/mm $\left.{ }^{3}\right)$ & $1.65 \pm 0.89$ & $1.74 \pm 0.69$ & 0.171 \\
\hline INR & $1.07 \pm 0.34$ & $0.99 \pm 0.17$ & $0.007 *$ \\
\hline Urine albumin & $1.74 \pm 0.78$ & $1.37 \pm 0.6$ & $<0.001 *$ \\
\hline Urea (mg/dl) & $33.49 \pm 28.42$ & $27.07 \pm 9.43$ & $0.007 *$ \\
\hline S. Cr (mg/dl) & $0.95 \pm 0.88$ & $0.77 \pm 0.19$ & $0.012 *$ \\
\hline SGOT (U/I) & $93.55 \pm 260.73$ & $47.39 \pm 64.83$ & $0.026^{*}$ \\
\hline SGPT (U/I) & $81.79 \pm 164.79$ & $53.75 \pm 65.56$ & $0.033^{*}$ \\
\hline T. Bil (mg/dl) & $0.87 \pm 1.3$ & $0.67 \pm 0.3$ & $0.044^{*}$ \\
\hline Uric acid (mg/dl) & $6.32 \pm 1.95$ & $5.82 \pm 1.47$ & 0.078 \\
\hline LDH (U/l) & $548.77 \pm 359.43$ & $363.09 \pm 195.56$ & $<0.001 *$ \\
\hline T. Prt (mg/dl) & $5.96 \pm 1.18$ & $6.04 \pm 1.31$ & 0.344 \\
\hline
\end{tabular}

SBP-systolic blood pressure, DBP-diastolic blood pressure, MAP-mean arterial pressure, mmHg-millimeter of mercury, $\mathrm{SpO}_{2}-$ saturation of peripheral oxygen, Hb-Haemoglobin, Hct-Haematocrit, Plt-platelet, INR-Internationalised normal ratio, SCr- Serum creatinine, SGOT-Serum glutamate oxaloacetate transaminase, SGPT-Serum glutamate pyruvate transaminase, T. Bil-total bilirubin, LDH-Lactate dehydrogenase, T Prt-Total protein, mg/dl-milligram per decilitre, U/l- unit per litre.*P value significant $(<0.05)$.

\section{Combined adverse (both maternal and perinatal) outcome}

Adverse combined outcome (maternal and perinatal) was present in $374(75 \%)$ of study population and $123(25 \%)$ had normal pregnancy outcome. Most common presenting symptom in combined adverse outcome was headache 171 (34.40), similar results seen in maternal and perinatal outcome.

\section{Multivariate analysis}

Logistic regression (for adverse maternal outcome)

A multivariate logistic regression using forward logistic regression model selection criteria was used to identify the best set of predictors for predicting adverse maternal outcomes in patients with preeclampsia. The best model was obtained in 6 steps wherein the explanatory power increased from $13.1 \%$ to $23.2 \%$. Significant predictors for predicting adverse maternal outcomes were identified as headache, chest pain/dyspnoea, $\mathrm{SpO}_{2}, \mathrm{Hb}$, INR and SGPT (Figure 1).

For predicting adverse perinatal outcomes in women with preeclampsia, the best model was obtained in 4 steps wherein the explanatory power increased from $5.1 \%$ to $9.8 \%$. Significant predictors for adverse perinatal outcomes identified were gestational hypertension in present pregnancy, headache, SBP and urine albumin (Figure 2).

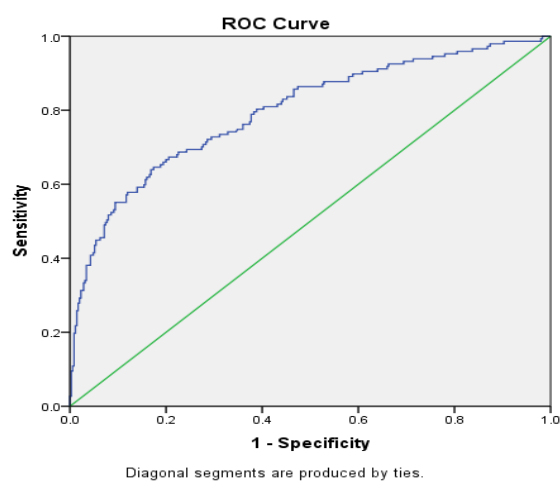

Figure 1: ROC curve (for adverse maternal outcome). AUC ROC 0.796, 95\% CI (0.751-0.841).

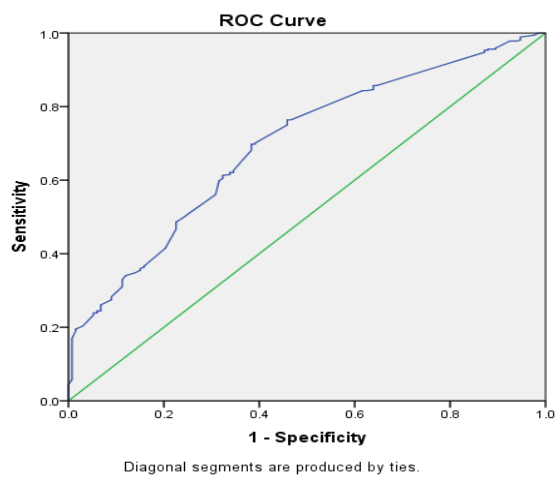

Figure 2: ROC curve (for adverse perinatal outcome). AUC ROC 0.695, 95\% CI (0.644-0.746). 
For predicting both adverse maternal and perinatal outcome, the best model was obtained in 5 steps wherein the explanatory power increased from $5 \%$ to $11.4 \%$. Significant predictors for predicting both adverse maternal and perinatal outcome were identified as gestational hypertension, headache, SBP, $\mathrm{Hb}$, urine albumin (Figure 3).

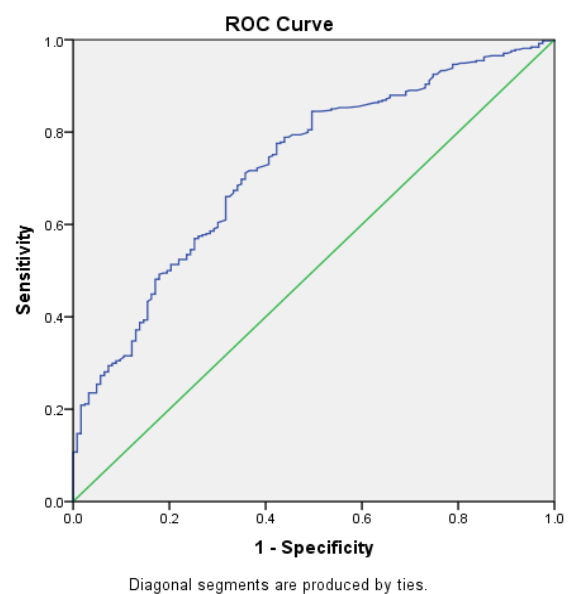

Figure 3: ROC curve (for both adverse maternal and perinatal outcome) AUC ROC 0.724, 95\% CI (0.6740.775).

\section{DISCUSSION}

It has been observed that preeclampsia is more common in young or elderly primigravida and it is reported that maternal age $>35$ years is significantly associated with preeclampsia. ${ }^{5}$ This is due to progressive vascular damage that occurs with aging. However, in the present study, the mean age of women with preeclampsia was $26.82 \pm 4.48$ years, probably because in this region the age of primigravida is mostly between $20-30$ years. In the present study both the patients with preeclampsia with adverse outcome and with normal pregnancy outcome were comparable with respect to age. Payne et al in analysis of PIERS study reported mean age to be 24.8 to 31 years. $^{6}$ Majority of study population in the present study had 10 years of education and belong to upper lower socioeconomic status. Similar results were seen in a study by Silva et al done in 3547 women which shows women with low socioeconomic status were more likely to develop preeclampsia than women with high educational level. ${ }^{7}$ Mean gestational age at delivery in the present study population was $35.6 \pm 3.76$ weeks. Similar results seen in study by Agarwal et al. ${ }^{8}$

Out of 497 study population, 147 (30\%) developed adverse maternal outcome, $364(73 \%)$ developed adverse perinatal outcome and $374(75 \%)$ developed adverse combined (both maternal and perinatal outcome). In the present study, the most common maternal complication was alteration in haematological system which were present in $75(15.09 \%)$ followed by neurological complication-45 (9.05\%), placental abruption-20 (4.02\%) and pulmonary oedema $9(1.81 \%)$ respectively in patients with preeclampsia. PIERS model was also dominated by haematological complications, such as transfusion of any blood product or low platelet count, which contributed to about half of all complications. ${ }^{6}$ Transfusion of blood products scored the lowest for clinical importance in a study by Thangaratinum et al on early-onset preeclampsia. $^{9}$

Most common perinatal complications observed in the present study was small for gestational age in 267 $(54.37 \%)$ followed by prematurity in $262(53.36 \%)$, still birth in $68(13.84 \%)$ and respiratory distress syndrome in $59(12.01 \%)$ of study population. Full PIERS model does not include fetal parameters such as intrauterine growth restriction or fetal death which have been found to be independent risks and only maternal outcomes are assessed. $^{6}$

Mean birth weight of babies with adverse outcome was $1.85 \pm 0.61 \mathrm{~kg}$ which was $1.1 \mathrm{~kg}$ less than the mean birth weight of babies with normal perinatal outcomes. Similar results seen in full PIERS model in which mean birth weight of babies with adverse perinatal outcome was $1.91 \pm 0.71 \mathrm{~kg}^{6}$

Among 308 (61.91\%) multigravidas with preeclampsia in present pregnancy in the present study, only $12(3.89 \%)$ had a hypertensive disorder in their previous pregnancies. This percentage was quite low, probably due to unsupervised previous pregnancies in the present study population however, women who had hypertension or preeclampsia in a prior pregnancy have a higher than normal risk of developing it again in a subsequent pregnancy. GDM and preeclampsia share many risk factors, including advanced maternal age, nulliparity, multifetal pregnancies, non-white race/ethnicity and prepregnancy obesity. ${ }^{10}$ Contrasting results seen in the present study. Only $28(5.63 \%)$ study population had associated gestational diabetes mellitus in present pregnancy due to unsupervised previous pregnancies of the study population.

In study population, most of them who developed adverse outcome were likely to receive magnesium sulphate during their clinical course and deliver babies earlier and of low birth weight. In study population, 304 (61.17\%) received magnesium sulphate (prophylactic and therapeutic), 492 (98.99\%) received antihypertensives, $161(32.39 \%)$ received steroids (for fetal lung maturity), similar results seen in full PIERS model. ${ }^{6}$

More than half of women in study population experienced at least one symptom, headache was present in $171(34.44 \%)$, over one third of study population in which, $102(20.52 \%)$ patients who developed headache had normal pregnancy outcome which could be explained as migraine headache and preeclampsia are both characterised by disordered reactivity and abnormal platelet activity, hence this similarity explains the high 
incidence of headache among women with preeclampsia. Similar results were seen in a study by Martin et al in which $34 \%$ of study population experienced headache a far greater number than who eventually suffered a morbid event. $^{11}$

Nausea and vomiting were the next most commonly experienced symptoms and it is statistically important in prediction of adverse maternal outcome. Yen et al in-sub analysis of PIERS study done in 2023 women, 52\% women experienced at least one symptom with $5.2 \%$ and $5.3 \%$ respectively experiencing an adverse maternal and perinatal outcome. ${ }^{12}$

In the present study, $\mathrm{SpO}_{2} \leq 96.90 \pm 2.60$ successfully predicted adverse maternal outcome $(\mathrm{p}<0.05)$. Adverse maternal outcome increases with the decrease in $\mathrm{SpO}_{2}$ value. This explains that reduced pulmonary function measured as decreased $\mathrm{SpO}_{2}$ is a marker of severe disease in general and is not specific for pulmonary oedema alone. In a study by Milliman et al using the data from PIERS multicentre study found that $\mathrm{SpO}_{2}<93 \%$ confers a particular risk and successfully predicts adverse maternal outcome. $^{13}$

In the present study, BP measurements (SBP, DBP, MAP) came out to be good predictor for predicting adverse maternal and perinatal outcome. In a study done by Cnossen et al reported that MAP is better predictor for pre-eclampsia than SBP, DBP or increased BP. ${ }^{14}$

In the present study, the authors tried to find out the association between investigative parameters with adverse outcomes. Urine albumin (proteinuria by dipstick method) was found to be statistically significant in the present study for both maternal and perinatal and combined (both maternal \pm perinatal) adverse outcome. Urine albumin (by dipstick method) came out to be independent predictor and one of best biochemical predictor variable for prediction of adverse perinatal and combined (maternal \pm perinatal) adverse outcome. In study done by Chan et al they demonstrated that no level of proteinuria could be used to predict outcomes and is a poor indicator of either maternal or perinatal outcome. ${ }^{15}$

Pre-eclampsia integrated estimate of risk (PIERS) study, a prospective study of 2023 women with preeclampsia, showed that none of dipstick, spot protein-creatinine ratio, or the 24 hours urine test predicted maternal or foetal outcomes accurately (AUC ROC $<0.7$ in all cases). ${ }^{6}$

In the present study fall in platelet count doesn't came out be independent predictor but was statistically significant ( $\mathrm{p}$ value $<0.05$ ) in predicting adverse maternal outcome. Platelet count of $<1.5 \mathrm{lakh} / \mathrm{cumm}$ was significantly associated with adverse maternal outcome in a study by Srivastava et al. ${ }^{16}$ Similar results were seen in the study by Agrawal et al. ${ }^{8}$
In the present study, all analytes of hepatic function like SGPT, SGOT, lactate dehydrogenase, bilirubin, albumin, and international normalized prothrombin time ratio came out to be statistically significant in prediction of adverse maternal and perinatal outcome but only SGPT (serum glutamate pyruvate transaminase) came out be independent variable in predicting adverse maternal outcome when logistic regression was applied.

Some authors suggest that analytes such as LDH, bilirubin, and possibly AST may prove to be more predictive because they reflect multiple organ dysfunction. The LDH level reflects haemolytic cell damage and hepatic dysfunction, the bilirubin level reflects both haemolysis and hepatic dysfunction, and the AST level reflects tissue damage and hepatic dysfunction. In a study by Kozic et al adverse maternal outcomes were more common in women with abnormal AST, ALT, $\mathrm{LDH}$, total bilirubin, and INR results $(\mathrm{p}<0.05) .{ }^{17}$ Present study found that, uric acid and creatinine to be statistically significant but are not found to be independent predictors for maternal and perinatal outcome.

A systematic review on uric acid and preeclampsia found that, in women with preeclampsia, a positive test result of uric acid greater than or equal to a 350-mmol/l threshold predicted eclampsia with a pooled logistic regression of 2.1 (95\% CI 1.4-3.5), while a negative test result had a pooled logistic regression of 0.38 (95\% CI $0.18-0.81$ ). The review concluded that serum uric acid is a poor predictor of maternal and fetal complications in women with preeclampsia. ${ }^{18}$ However, Hawkins et al. in a later study concluded that the risk of adverse maternal outcome (OR 2.0; 95\%, CI 1.6-2.4) and adverse fetal outcome (OR 1.8; 95\%, CI 1.5-2.1) increased with increasing concentration of uric acid. ${ }^{19}$ In a study on by Agrawal et al, serum creatinine was found to be an independent predictor of adverse maternal outcome. ${ }^{8}$

In the present study, after applying logistic regression, we identified best predictor variables for maternal, perinatal and combined (both maternal and perinatal) adverse outcome. For maternal outcome, best predictors were calculated in 6 steps wherein the explanatory power increased from 13.1 to $23.2 \%$. Best predictors for maternal adverse outcome are headache, chest pain/dyspnoea, $\mathrm{SpO}_{2}$, haemoglobin, international normalised ratio, SGPT. In full PIERS study also chest pain and $\mathrm{SpO}_{2}$ were found to be best predictor but other four variables were different.

For perinatal outcome best predictors were calculated in 4 steps wherein explanatory power increased from 5.1 to $9.8 \%$, best predictors were-gestational hypertension, headache, systolic blood pressure, urine albumin by dipstick method.

For combined (both maternal and perinatal), best predictors were calculated in 5 steps wherein explanatory 
power increased from 5.0 to $11.4 \%$, best predictors were gestational hypertension, headache, systolic blood pressure, haemoglobin, urine albumin by dipstick method.

\section{CONCLUSION}

Thus, from this study we concluded that simple clinical and biochemical parameters maintained its utility as a prediction tool to be used as a rule-in test for adverse maternal, perinatal and combined (both maternal and perinatal) outcomes and recommended timely referral to a higher centre in reducing the morbidity and mortality associated with this condition.

Funding: No funding sources

Conflict of interest: None declared

Ethical approval: The study was approved by the Institutional Ethics Committee

\section{REFERENCES}

1. Sibai B, Dekker G, Kupferminc M. Pre-eclampsia. Lancet. 2005;365:785-99.

2. Menzies J, Magee L, Li J, Ying C, Nab M, Ruihua Y et al. Instituting surveillance guidelines and adverse outcomes in preeclampsia. Am Coll Obstet Gynaecol. 2007;110:121-7.

3. Androes P, Kalbaugh A, Taylor S, Blackhurst D, McClary E, Gray J et al. Does a standardization tool to direct invasive therapy for symptomatic lower extremity peripheral arterial disease improve outcomes. Vasc Surg. 2010;40:907-15.

4. North A, Taylor S, Schellenberg C. Evaluation of a definition of pre-eclampsia. Br J Obstet Gynaecol. 2009;106:767-73.

5. Yun W, Tom T, Thomas A, Tore H. The impact of advanced maternal age and parity on obstetric and perinatal outcomes in singleton gestations. Arch Gynecol Obstet. 2011;284:31-7.

6. Payne B, Hodgson S, Hutcheon J, Joseph K, Li J, Lee $\mathrm{T}$ et al. Performance of the full PIERS model in predicting adverse maternal outcomes in preeclampsia using patient data from the PIERS (Preeclampsia Integrated Estimate of Risk) cohort, collected on admission. $\mathrm{Br} \mathrm{J}$ Obstet Gynaecol. 2013;120:113-8.

7. Silva LM, Coolman M, Steegers EA, Jaddoe VW, Moll HA, Hofman A et al. Low socioeconomic status is a risk factor for preeclampsia: The Generation R Study. J Hypertens. 2008;26:1200-8.

8. Agarwal S, Maitra N. Prediction of adverse maternal outcomes in preeclampsia using a risk prediction model. J Obstet Gynaecol India. 2016;66:104-11.
9. Thangaratinam S, Coomarasamy A, Sharp S, Mahony OF, Obrien S. Tests for predicting complications of preeclampsia: a protocol for systematic reviews. BMC Pregnancy Childbirth. 2008;8:38.

10. Mudd LM, Owe KM, Mottola MF, Pivarnik JM. Health benefits of physical activity during pregnancy: an international perspective. Med Sci Sports Exerc. 2013;45:268-77.

11. Martin JN Jr, May WL, Magann EF, Terrone DA, Rinehart BK, Blake PG. Early risk assessment of severe preeclampsia: admission battery of symptoms and laboratory tests to predict likelihood of subsequent significant maternal morbidity. Am J Obstet Gynecol. 1999;180:1407-14.

12. Yen TW, Payne B, Qu Z, Hutcheon JA, Lee T, Magee LA et al. Using clinical symptoms to predict adverse maternal and perinatal outcomes in women with preeclampsia: data from the PIERS (preeclampsia integrated estimate of risk) study. J Obstet Gynaecol Can. 2011;33:803-9.

13. Millman A, Payne B, von Dadelszen P, PIERS (Preeclampsia Integrated Estimate of RiSk) Study Group. Oxygen saturation as a predictor of outcomes in women with pre-eclampsia. Pregnancy Hypertens. 2010;1:58.

14. Cnossen SJ, Vollebregt KC, deVrieze N, Franx A, Khan KS, Mol BW et al. Accuracy of mean arterial pressure and blood pressure measurements in predicting pre-eclampsia: Systematic review and meta-analysis. Br Med J.2008;336:1117-20.

15. Chan P, Brown M, Simpson JM, Davis G. Proteinuria in pre-eclampsia: how much matters? $\mathrm{Br}$ J Obstet Gynaecol. 2005;112:280-5.

16. Srivastava S, Parihar BC, Jain N. PIERS calculatorpredicting adverse maternal outcome in preeclampsia. Int $\mathrm{J}$ Reprod Contracept Obstet Gynecol. 2017;6:1200-5.

17. Kozic JR, Benton SJ, Hutcheon JA, Payne BA, Magee LA, von Dadelszen $\mathrm{P}$ et al. Abnormal liver function tests as predictors of adverse maternal outcomes in women with preeclampsia. J Obstet Gynaecol Can. 2011;33:995-1004.

18. Thangaratinam S, Ismail K, Sharp S. Accuracy of serum uric acid in predicting complications of preeclampsia: a systematic review. $\mathrm{Br} \mathrm{J}$ Obstet Gynaeco.1 2006;113:369-78.

19. Hawkins T, Roberts J, Mangos G. Plasma uric acid remains a marker of poor outcome in hypertensive pregnancy: a retrospective cohort study. $\mathrm{Br} \mathrm{J}$ Obstet Gynaecol. 2012;119:484-92.

Cite this article as: Tongaria K, Kumar A, Kaur S. Prediction of adverse effects of preeclampsia. Int J Reprod Contracept Obstet Gynecol 2020;9:4420-7. 